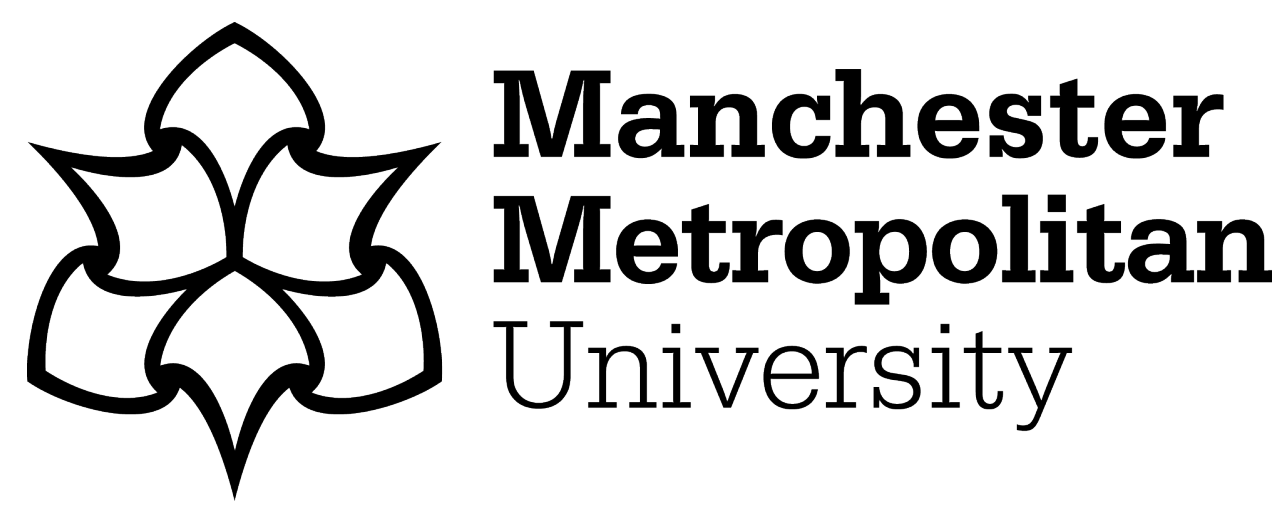

Upadhyay, Arvind, Mukhuty, Sumona ORCID logoORCID: https://orcid.org/0000-0003-2408-7123, Kumar, Vikas and Kazancoglu, Yigit (2021) Blockchain technology and the circular economy: Implications for sustainability and social responsibility. Journal of Cleaner Production, 293. p. 126130. ISSN 0959-6526

Downloaded from: https://e-space.mmu.ac.uk/627269/

Version: Accepted Version

Publisher: Elsevier BV

DOI: https://doi.org/10.1016/j.jclepro.2021.126130

Usage rights: Creative Commons: Attribution-Noncommercial-No Derivative Works 4.0

Please cite the published version 


\section{Blockchain Technology and the Circular Economy: Implications for Sustainability and Social Responsibility}

\section{ABSTRACT}

Blockchain technology is a revolutionary new protocol for sharing and updating information by linking ledgers or databases in a decentralised, peer-to-peer, open-access network. Blockchain is designed to ensure the data is stored and updated in a secure, tamper-proof and irreversible way. Despite being in its nascent stages, the blockchain research is developing rapidly in different fields, making it imperative to capture the ethical and sustainability implications of blockchain development and implementation. The circular economy also focuses on enhancing sustainability and social responsibility, alongside economic growth. In this article, we critically review blockchain technology's current and potential contribution to the circular economy through the lens of sustainability and social responsibility. This paper contributes to the Industry 4.0 literature by identifying, collating and organising the disparate research on blockchain, with a critical focus on its positive impact and potential repercussions for the ethics agenda. Within this narrative review, we argue and highlight the extant and potential alignment of blockchain with circular economy. Our findings show that blockchain technology can contribute to the circular economy by helping to reduce transaction costs, enhance performance and communication along the supply chain, ensure human rights protection, enhance healthcare patient confidentiality and welfare, and reduce carbon footprint. We also evaluate the challenges to blockchain implementation for circular economy, in terms of trust, illegal activities, potential for hacking and the need to address these through suitable legislation and policy development. Furthermore, we acknowledge the potential upfront costs involved in implementing blockchain technology, although we observe that the benefits are likely to exceed the challenges. We conclude this article with recommendations for future research in this field.

\section{Keywords: Blockchain, Circular Economy, Sustainability, Social Responsibility, Industry 4.0}

\section{Introduction}

Industry 4.0 or the 'Fourth Industrial Revolution' as introduced by the German federal government in 2011 (Lasi et al., 2014), places high emphasis on enhancing industrial capabilities through the intensification of technological applications and digitalisation (Luthra et al., 2020). Industry 4.0 embraces smart systems harnessed through information technology (IT) (de Sousa Jabbour et al., 2018a) and encompasses digital and virtual technologies which are product and service focused (de Sousa Jabbour et al., 2018b), facilitate real-time data exchange and updates, and are interconnected. Lu (2017) captures this concisely, stating that Industry 4.0 features "digitization, optimization, and customization of production; automation and adaptation; human-machine interaction ( $\mathrm{HMI}$ ); value-added services and businesses, and automatic data exchange and 
communication" (Lu, 2017). In other words, Industry 4.0 aims to bring about the development and synthesis of digital, physical and biological technologies (Li et al., 2017) through software and programming expertise.

To achieve this fusion, digital technology currently remains the core driving force of Industry 4.0 (Li et al., 2017). A key aim and feature of Industry 4.0 is the facilitation of inter-connection between machines, digital devices, transactions, and various stakeholders including suppliers, buyers, and customers (de Sousa Jabbour et al., 2018b), in real-time. The 'Internet of Things (IoT)' has made this interconnected communication possible to an extent. Furthermore, a revolutionary new technology, which promises to make this possible in a more secure fashion, is Blockchain technology.

Blockchain technology may be defined as an online, open-source distributed ledger where transactions between different stakeholders can be recorded and updated simultaneously and in real-time (Iansiti \& Lakhani, 2017). Blockchain is designed so that these transaction records can be authenticated in a reliable way and the records are irreversible (lansiti \& Lakhani, 2017). Blockchain is a peer-to-peer system that eschews the role and power of centralised control over transactions, their record-keeping and access to these records. Thereby it can facilitate the fusion of transactions, stakeholders as well as machines, a key feature and aspiration of Industry 4.0. Industry 4.0's loT entails communication and interconnectivity between things like electronic devices, smart devices including smartphones, and transportation systems using unique codes (de Sousa Jabbour et al., 2018b). Blockchain has the potential to underpin and bring security to this key Industry 4.0 aspiration of loT. Blockchain can also facilitate automation of transactions in a permanent and verifiable manner (Iansiti \& Lakhani, 2017), helping to save time and resources and thereby eliminating inefficiencies or waste.

Industry 4.0 can also be linked to the aims of the Circular Economy (de Sousa Jabbour et al., 2018b), which is to achieve a regenerative, closing the loop economy with minimal waste (Geissdoerfer et al., 2017), aspiring to detangle environmental pressure from economic enhancement and harmonise between the environment, economy and society (Ghisellini et al., 2016). Circular Economy seeks cleaner production arrangements, adoption of renewable technology and know-how, and development of appropriate policies and tools (Ghisellini et al., 2016). Blockchain by virtue of its make-up as an open-source, peer-to-peer, distributed ledger system and automation capabilities; has the potential to create cleaner economic transactional processes and help achieve the muchneeded balance and harmony between the environment, economy and society. Effectively the implementation of a Circular Economy can also contribute to the Green Economy agenda (Ghisellini et al., 2016) through the development and diversified implementation of blockchain technology. 
Arguably, the underlying premise of the Circular Economy is the ethical business agenda of cleaner production (Hens et al., 2018), sustainability (Geissdoerfer et al., 2017) and social responsibility (Esken et al., 2018). This encompasses both service and manufacturing industries in the economy. Blockchain technology as a powerful distributed ledger tool of secure interconnectivity has the prowess to facilitate cleaner production of goods and services and address the ethical agenda of business development.

While blockchain technology was first developed and introduced in 2008 (Xu et al., 2019); it has taken several years for different industries to recognise its potential value and utility in exponentially enhancing efficiencies and reducing waste. Despite being in its nascent stages, the associated research is developing rapidly in different fields, making it imperative to capture the ethical implications of blockchain development and implementation and bridge this gap in the literature.

Therefore, the aim of this article is to critically review blockchain technology's current and potential contribution to the circular economy through the lens of sustainability and social responsibility. This paper contributes to the Industry 4.0 literature by identifying, collating and organising the disparate research on blockchain, with a critical focus on its positive impact and potential repercussions for the ethics agenda.

This study is structured as follows: The following section explains the methodology adopted in conducting this research. The subsequent section reviews the concept of blockchain, circular economy and argues the interlink between the two. This is followed by an evaluative review of blockchain and its application in different sectors, in relation to sustainability and wider social responsibility implications. Within this review, we argue and highlight the extant and potential alignment of blockchain with circular economy. The final section encompasses the key conclusions, and provides recommendations for future research in this field.

\section{Research methodology}

We conduct this research by adopting a narrative and integrative literature review approach as advocated by Torraco (2005). An integrative literature review is considered suitable where the subject matter is new and needs further exploration (Torraco, 2005). There is a need for this review as blockchain is a new technology, which is rapidly gaining implementation interest in different sectors. Organisations are enthusiastic to capitalise on cost and time efficiencies. However, there is an increased need and interest to balance this with sustainability and social responsibility imperatives embodied in the cleaner production and circular economy agenda, an example of which is the UN sustainable development goals (SDGs) towards 2030 (United Nations, 2015). The 
exponential level and speed of investment in blockchain implementation experiments make it crucial to investigate blockchain technology in terms of how it can make a beneficial contribution to sustainability and social responsibility, while raising awareness of the potential ethical drawbacks of blockchain. To accomplish this, we need to draw linkages between different bodies of literature; achievable through an integrative study (MacInnis, 2011; Torraco, 2005). Through this review, we aim to generate new perspectives on the implication of blockchain technology on the ethical business agenda of industry 4.0 .

We draw upon the combined literature of blockchain technology and its applications in business, sustainability and social responsibility to capture the current influence of blockchain and develop an analysis on its potential implications. In doing so, we also analyse relevant literature on the circular economy, a core aspect of which is sustainability. We critically synthesise segregated fields of literature to develop a combined conceptual analysis leading to new perspectives. The rationale for this approach becomes apparent through a review of the literature on blockchain, followed by circular economy and sustainability, leading to the arguments illustrating how blockchain can augment as well as hinder the sustainability and social responsibility imperative.

In this study, we included articles focusing on blockchain in the field of business and its applications; as opposed to articles on computational language and algorithms. Furthermore, we included articles on sustainability and social responsibility based on whether there was a link with industry 4.0, circular economy, or blockchain itself. To establish the relevance of the articles, we included articles where the search terms were evident in the title and/or the abstract. We conducted initial article searches on academic search engines including EBSCO Business Source Premier, Web of Science, Science Direct and Cross Ref. Thereafter, we adopted a snowballing approach and identified suitable articles from citations of the articles retrieved from exploration on academic search engines. We discovered that the majority of articles on blockchain were published 2015 onwards with a surge in such articles between 2017 and 2020. The bulk of the articles on circular economy were published post 2010. Despite the apparently short timeline, research in this field is increasing at an exponential rate, making this review a timely one. Due to the nascent nature of blockchain research, this review was not restricted to 'top journals' only, although it includes a high number of top journals.

Sustainability and social responsibility are relatively established topics, but blockchain is a novel invention, which is just over a decade old, and its implementation in different sectors is still gathering pace. In order to analyse the make-up and applications of blockchain in the context of 
sustainability and social responsibility, it is important to understand the technology, how it works and its core features. Thereafter we can evaluate its alignment with and implications for sustainability and social responsibility in the Circular Economy. Therefore, in the following sections, we commence with a conceptual review of blockchain technology, leading on to a synthesis of this literature with the circular economy, sustainability and social responsibility.

\section{Key Concepts: Blockchain technology and the Circular Economy}

\subsection{Blockchain Technology}

Blockchain technology was developed and first introduced by Satoshi Nakamoto (pseudonym), as an open distributed ledger tool for the use of cryptocurrencies like bitcoin (Nakamoto, 2008). Although scepticism towards its complexity initially stifled blockchain development, growing recognition of its advantages has led to burgeoning research on its applications. Blockchain has been hailed as both a foundational (Iansiti \& Lakhani, 2017) and disruptive technology (Jesse, 2018; Xu et al., 2019). The key attractive features of blockchain technology include its use as a distributed ledger, decentralised control, transparency, permanency of transactions, robust makeup preventing tamper or manipulation, and openness of accessibility (lansiti \& Lakhani, 2017; Xu et al., 2019). Blockchain technology development has now developed into three versions, Blockchain 1,.0, 2.0 and 3.0; aligning with different types of implementation.

Blockchain technology "handles blocks - uniquely identified, linked transaction records - in a chain" (Treleaven et al., 2017, p. 15). It functions, so that once a transaction is updated and authenticated by all the relevant nodes or key parties in the network, it is irreversible and cannot be over-written or re-sequenced. This makes blockchain a very secure distributed ledger and resultantly all parties have access to identical and correct records (Iansiti \& Lakhani, 2017; Moll \& Yigitbasioglu, 2019). Furthermore, peer-to-peer interconnected ledgers ensure that updates in any one ledger are visible and accessible to all. Furthermore, blockchain is a perpetually expanding, distributed yet shared ledger secured cryptographically, using digital fingerprints (Treleaven et al., 2017).

In order to add new blocks to the chain, cryptocurrency mining is adopted which entails deciphering a mathematical conundrum via large intricate computations, until the target value is reached. In the physical world, this is like using trial and error to arrive at the correct combination for a safe. Miners on the network compete to find blocks by solving the conundrum quickest; and add these to the chain. In return, they are rewarded in new cryptocurrency. Each block needs to contain a proof-ofwork, which is ratified by other nodes on the network. This ensures the security and tamper- 
resistance of the chain (Flint, 2014; Moll \& Yigitbasioglu, 2019). The system is set up so that there is a limit on the amount of cryptocurrency released into circulation. Blockchain, therefore is established on the principle of simultaneous cooperation and competition within the network (Narayan \& Tidström, 2020).

Blockchain 2.0 technology can also be employed to implement automation of transactions (lansiti \& Lakhani, 2017; Moll \& Yigitbasioglu, 2019), which can be set up via digital, self-executing 'smart contracts' (Xu et al., 2019). This concept was initiated by Ethereum, a blockchain platform; although the concept was first coined by Nick Szabo in 1993, but the lack of infrastructure prevented its implementation (Szabo, 1997). Smart contracts are defined by pre-specified terms, incorporating rules and penalties, set up on the blockchain employing a Turing-complete contracting language (Lai \& Lee Kuo Chen, 2017). This facilitates the setting-up and auto-execution of complex contracts (Moll \& Yigitbasioglu, 2019; Omohundro, 2014). These contract terms are openly visible to all nodes on the network or blockchain (Moll \& Yigitbasioglu, 2019). A smart contract is triggered when it receives data which is assessed in terms of the pre-defined contractual terms. If the pre-set conditions are met, the required outcome is generated i.e. completion of the transaction, otherwise, an error message is released, which is discernible to all nodes on the smart contract blockchain (Rozario \& Vasarhelyi, 2018). The diversified implementation of smart contracts in different fields also promises to lower the cost of transactions.

Blockchain technology was developed as a communication tool between different ledgers to upgrade digital currency transactions simultaneously and irreversibly. The primary application of blockchain being, recording the cryptocurrency bitcoin's usage and transactions. Since its initial application in cryptocurrencies and evolution to implement smart contracts, blockchain application has extended to the banking and finance sector (lansiti \& Lakhani, 2017) and accounting field (Moll \& Yigitbasioglu, 2019). Blockchain is subsequently being experimented with and researched in the health sector (Engelhardt, 2017; Radanović \& Likić, 2018), supply chain management (Saberi et al., 2019; Schmidt \& Wagner, 2019), marketing, smart technology schemes, insurance (Püttgen \& Kaulartz, 2017), patents and copyrights (O'Dair \& Beaven, 2017)and so on. What makes blockchain attractive to different sectors are the principles underpinning the technology, namely, peer-to-peer sharing, distributed storage capability, tamper-resistant security and the potential for secure automation.

\subsection{Circular Economy}


The concept of Circular Economy attempts to integrate economic activities with environmental welfare in a sustainable manner (Murray et al., 2017). The circular economy aims to depart from the practice of 'make, use, dispose' and encourages the cyclical implementation of processes and use of processes. It aims to restrict the depletion of natural resources and waste, through its cyclical use and embodies cleaner production through the three Rs 'reduce, reuse and recycle' (Murray et al., 2017), both in the manufacturing and service economy. Circular economy defines a new approach to addressing sustainability and social responsibility issues; paying particular attention to the social aspects of sustainability. It embraces a loop system of economy-environment collaborations creating a closed-loop economy (Mathews \& Tan, 2011). Within the loop, the circular economy aims to keep materials, products and outputs in circulation for the longest time, by preserving their value (Bocken et al., 2016). Alongside, economic growth while protecting the environment, Circular Economy also aims to create social equality (Xue et al., 2010). Murray et al. (2017) also highlighted the need for the Circular Economy agenda to focus on achieving social equality. In addition to being considered by some governments like China, industry and academics, the Circular Economy is being championed by think-tanks in the west like the Ellen MacArthur Foundation (MacArthur et al., 2015). Natural resources are finite and irreplaceable, while economic growth and job creation is still a necessity, hence there a need business models fostering sustainable living, production and consumption (Murray et al., 2017) both in the manufacturing and the service sector. Hence, increasingly, organisations are keen to adopt the circular economy concept in integrating sustainability into economic activity (Bocken et al., 2016). Seeking to generate economic value in tandem with sustainability and social welfare is leading firms towards co-creation of sustainable market value through transformative business strategies (Loorbach \& Wijsman, 2013). In the context of circular economy, Spring and Araujo (2017) propose designing products as distributed networks rather than a unitary product; with service-value opportunities at various transition points of a product life-cycle (Spring \& Araujo, 2017).

The Ellen MacArthur Foundation, dedicated to holistic development and implementation of the circular economy proposes three principles for circular value creation (Ellen MacArthur Foundation, 2015). First, preserving and enhancing finite supply of natural resources through dematerialisation and virtual delivery of utility, and embracing renewable technologies and processes. Second, optimisation of products and processes through recycling, refurbishment and remanufacturing to regenerate resources and recirculate them without diminishing value. Third, reducing damage, designing out waste and use of renewable and resilient resources for economic growth (Ellen MacArthur Foundation, 2015). They also advocate the ReSOLVE framework to nurture the circular 
economy: regeneration, sharing, optimisation, loop, virtualisation and exchange. Regeneration entails renewals and restoration of ecosystems. Sharing focuses on reusing, upgrading and sharing asset usage. Optimisation includes enhanced performance/efficiency, waste removal and big data usage, remote sensing and automation. Loop refers to recycling, remanufacturing, and biochemical extraction from organic waste so outputs act as inputs in the economy. Virtualisation encourages dematerialisation in the form of e-books, digital music and online marketplaces. Finally, exchange entails adopting new models of services and business and applying new technological inventions like 3D printing and blockchain platforms (Ellen MacArthur Foundation, 2015). Arguably, this circular economy framework of sustainability can be achieved significantly through digitalisation and particularly blockchain technology. The following section argues the link between blockchain technology and the circular economy.

\subsection{Blockchain and Circular Economy Linkage}

Increasingly digitalisation is making it possible to implement the Circular Economy vision of being regenerative and restorative. These particularly include industry 4.0 technology like artificial intelligence, big data analysis, cloud computing, internet of things (IOT) and 3D printing (de Sousa Jabbour et al., 2018; Despeisse et al., 2017; Ellen MacArthur Foundation, 2019; Pagoropoulos et al., 2017). The advent of blockchain technology has provided a secure, interconnected yet distributed system to facilitate tamper-proof and green implementation of loT (Jiang et al., 2019), 3D manufacturing (Vatankhah Barenji et al., 2020), additive manufacturing (Mandolla et al., 2019), food supply (Ellen MacArthur Foundation, 2019), health care (De Aguiar et al., 2020) and so on. Blockchain can be applied to develop and coordinate novel circular economy networks (Narayan \& Tidström, 2020). In relation to the ReSOLVE framework (Ellen MacArthur Foundation, 2015) presented in the previous section, blockchain platforms can effectively cater to the elements of sharing, optimisation, virtualisation and particularly exchange. Thereby opening up a treasure trove of regenerative digital possibilities for the circular economy goal of sustainability.

In the context of the circular economy, we may consider blockchain as a social technology or tool for coordination. It helps to connect and coordinate multiple distributed databases (Swan, 2015), which can be all be updated simultaneously and accessible to all parties. The circular economy can also benefit from simultaneous cooperation and competition within the loop, adopting a decentralised principle of value creation and circulation as opposed to value creation and value appropriation (Narayan \& Tidström, 2020). This is a core feature of the blockchain technology and can aid in 
achieving the above circular economy outcomes through its principles of decentralisation, distributiveness, and tamper-resistance (Iansiti \& Lakhani, 2017; Narayan \& Tidström, 2020) coupled with its functionality for smart contracts (Catalini \& Gans, 2016) and tokenisation (Narayan \& Tidström, 2020).

Sustainability and wider social responsibility imperatives are achievable through business models facilitating decentralisation and circulatory value creation in products and services. The circular economy aim is to minimise or eliminate waste (Schulte, 2013), which can be facilitated by transforming product/services end-of-life into restorative value by enhancing longevity or deciphering new forms of consumption (Sousa-Zomer et al., 2017), thereby keeping them in sustainable circulation. Furthermore, some authors encourage linking the conceptualising of the circular economy to systems thinking, with a business model considering the wider network of stakeholders to ensure the cyclical life cycle in designing products and services (Murray et al., 2017; Narayan \& Tidström, 2020). Here-in blockchain technology can significantly contribute by providing the underpinning technology, linking complex networks and databases with built-in designs and configurations to update all connected databases simultaneously, irreversibly and facilitate automation where needed. For the success of such innovation, different firms on the network or chain, need to embrace the new technology and reconfigure as well (Geissdoerfer et al., 2017; Narayan \& Tidström, 2019). The innovative technology of blockchain protocol is being considered a strong driving force for digital economy (N. Upadhyay, 2020). The following section evaluates the different current and potential applications of blockchain protocol in various sectors and their implications for the circular economy aims of sustainability and social responsibility.

\section{Discussion of Blockchain and its Applications: Sustainability and Social Responsibility} implications for the Circular Economy

We have already established that sustainability is a core focus of the circular economy. Sustainability, in turn, subsumes three core interlinked pillars in the form of economic, environmental and social factors (Purvis et al., 2019). These pillars are also embedded in the UN Sustainable Development Goals (United Nations, 2012) towards 2030; emphasising economic development which is environmentally conscious, sustainable and socially responsible (Purvis et al., 2019). Industry 4.0 investment in digitalisation provides immense opportunities to achieve sustainable economic growth while preserving the environment and being socially responsible (Beier et al., 2020). One of the most potent digital developments in this regard, is blockchain protocol. In 
this section, we analyse how blockchain protocol and its applications in different sectors, can benefit and challenge the circular economy through sustainability and social responsibility considerations.

As blockchain was introduced as a distributed ledger to record the cryptocurrency bitcoin transactions, most naturally the finance and accounting sectors were pioneers in testing its wider application. Various banks started experimenting with private blockchain to replace paper-based and manual recording and authentication of transactions, especially in the context of financial trading cross-border transactions and foreign exchange (Iansiti \& Lakhani, 2017). Use of blockchain and smart contracts is heralding transformations in the initiation, processing, recording, resolution, audit and reporting of transactions (Schmitz \& Leoni, 2019). For example, smart contracts facilitate automating transactional payments by linking with GPS functionality and updating activities at different locations (Iansiti \& Lakhani, 2017). These systems thereby contribute to social responsibility by preventing fraudulent transactions. Furthermore, there is an opportunity for new financial models to move towards more sustainable investments contributing to the economy (Schulte, 2013). Blockchain protocol can help raise venture capital in a more equitable manner through crowdfunding and making access to finances fairer for all sections of society (Dierksmeier \& Seele, 2020; Greenberg \& Mollick, 2017).

Blockchain technology can be very attractive in supply chain management (Venkatesh et al., 2020). Supply chains are perpetually aspiring to reduce waste and enhance efficiencies (Upadhyay et al., In Press). Using blockchain technology to link the distributed ledgers, databases and stakeholders in the entire supply chain can enhance efficiencies and ensure savings in cost as well as time. Long and complex supply chains can be monitored with relative ease and efficiency by recording key data in the blockchain during the journey of the product from raw material source to manufacturer to customer (Xu et al., 2019). In this manner, product-quality and cleaner production is can be ensured, by transcending local and global boundaries. For example, in diamond manufacturing, using blockchain, the gems can now be tracked through the blockchain from the source mines to the final consumer (lansiti \& Lakhani, 2017). Blockchain can thereby align supply chains with circular economy sustainability through traceability and transparency (Ellen MacArthur Foundation, 2019) and reduce hard copy usage, fuel usage, encourage energy renewal and substantially reduce waste in supply chains. As blockchain is irreversible, the records of resources, updates and stakeholders can help generate cyclical processes for supply chain management. Furthermore, blockchain records help monitor and proof to the customer, the environmental sustainability of products manufactured. It also helps to ensure and assure the customers that child labour was not used and human rights were protected in the production and supply chain process (Dierksmeier \& Seele, 2020). Thus, 
through collaboration and knowledge sharing in the blockchain, we can achieve sustainability and social responsibility goals and foster a circular economy.

Healthcare is another important area where blockchain protocol and smart contracts are gaining momentum through permissioned blockchain (Engelhardt, 2017). Here, every blockchain is encrypted maintaining privacy as different parties on the chain can only access information they have the right to access through cryptographic codes. Accuracy, completeness and appropriate accessibility to healthcare records is a key concern in the health sector. From an ethical and social responsibility viewpoint, patient records technology needs to ensure patient confidentiality, and access by correct persons at the right times. The promise of privacy through an encrypted private blockchain, ability to interlink different databases for data completeness, inter-operability and costsavings by removal of third-party trust broker overhead requirements, has led to research and investment in how blockchain protocol can be implemented for patient data records maintenance and public health initiatives (Engelhardt, 2017; McGhin et al., 2019; Yue et al., 2016). The simultaneous ease of access for appropriate stakeholders and security offered by blockchain can also empower patients to own, share and co-create their own health data in a transparent manner (Schumacher, 2017; Yue et al., 2016), facilitating more patient control. Blockchain in healthcare can also create and take advantage of the smart contract functionality in dispensing and delivering public healthcare like updating and fulfilling prescriptions (Engelhardt, 2017). Use of blockchain in healthcare records management can also prevent prescription and healthcare fraud (Engelhardt, 2017; McGhin et al., 2019) and facilitate pharmaceutical recall of unsafe of defective products (Wu \& Lin, 2019). Thereby implementation of blockchain and its smart contract functionality in healthcare can make profound contributions to sustainable social responsibility in the economy.

Blockchain technology could potentially reduce carbon footprint. Blockchain protocol is being implemented in the automobile industry, not just to trace ownership but also develop secure automated driving technology. Blockchain can be employed to ensure tamper resistant vehicle security and protect automatic cars from being hacked (Dierksmeier \& Seele, 2020), a primary concern in using self-driving cars. Assurance of security will encourage consumption of these cars thereby reducing fuel usage and emissions. Similarly, Tang \& Tang (2019) proposed using blockchain technology to develop a distributed carbon ledger linking carbon asset management with carbon emissions trading schemes to effectively facilitate carbon accounting and control greenhouse gas emissions (Tang \& Tang, 2019). Adopting blockchain platform is also being advocated in developing smart cities with low carbon emission and green environment (Sun \& Zhang, 2020). 
While the future of blockchain in fostering the circular economy is bright, currently the path is not necessarily smooth. There are many challenges we need to be mindful of in implementing its widespread application. The paradox of simultaneous competition and cooperation (Bengtsson et al., 2016) within a blockchain in the circular economy, can give rise to issues of co-dependency, conflict and trust (Narayan \& Tidström, 2019). The automation feature of smart contracts have also led to concerns amongst the community of accountants, auditors and lawyers about the status quo and future nature of their roles (Iansiti \& Lakhani, 2017; Schmitz \& Leoni, 2019). The underpinning philosophy here is to move from the transactional to the relational; and design business models supported by professionals, focusing beyond profit-oriented markets towards the societal and social welfare (Narayan \& Tidström, 2020). Blockchain aims to elicit the trust of stakeholders through its decentralised and immutable algorithmic system (Engelhardt, 2017). Herein, blockchain acts as the trusted third part authenticator of transactions. As all the records are stored and updated with different nodes of the network, the onus of trust shifts from a third-party broker with information to all stakeholders on the network, thereby providing security and preventing tamper (Schmitz \& Leoni, 2019). Arguably, as trust in the system is developed through usage, blockchain technology will develop longevity and make valuable contributions to the circular economy through sustainability and social responsibility.

As stated earlier, the primary ethical attraction of blockchain is decentralisation of power and control (Dierksmeier \& Seele, 2020; Flint, 2014) providing an open access distributed ledger for all interested stakeholders. The decentralisation helps to eliminate power and information concentration with intermediaries like agents, brokers, lawyers, banks imposing surcharges for the information they provide. Eliminating dependence on intermediaries reduces time and financial costs to the contracting parties providing moral benefit (Dierksmeier \& Seele, 2020) and social welfare. However, this could also be seen as a double-edged sword, as blockchain supported cryptocurrencies could be adopted to fund criminal activities and avoid paying taxes (Flint, 2014). This could include increase in money laundering, trading on the dark web in illegal substances, flesh trading, human trafficking and other illegal activities (Seele, 2018); undetected by the authorities. The counter argument to this is that the same illegal activities can be and are in existence using regular currencies or cash.

A cautionary argument against blockchain is that complete security can never be a promise, where we have physical contact and multiple users; and despite anonymity and encryption a small risk of hacking remains (Xu et al., 2019). Infamously the bitcoin exchange system was hacked, which revealed that while blockchain protocol was robust in and of itself, security needed enhancing while linking separate systems connected to different stakeholders accessing the blockchain (lansiti \& 
Lakhani, 2017). However, it is argued that blockchain by virtue of being interlinked and interoperable has the ability to detect fraud in real time and thereby prevent or minimise it fraud (Wang \& Kogan, 2018) thereby helping firms contribute to social responsibility.

The complex nature of blockchain smart contracts, makes it extremely challenging for different parties to decipher the legality surrounding these contracts (Upadhyay, 2020). In developing governance and legislative tools, special attention needs to be paid to designing the rules, regulations and standards governing blockchain and smart contracts. Legislation and policy development of digitalisation needs to balance the ecological and economical needs, so that firms and investors can develop circular economy business models, which preserve the environment and yield economic returns on their investments (Ghisellini et al., 2016); using the technology. Proper governance and policing can also protect the general public from illegal activities of people attempting to implicate blockchain nodes in illegal activities and crypto-jacking (Dierksmeier \& Seele, 2020).

Implementing blockchain protocol and setting up smart-contracts needs significant upfront investment, to reap long-term sustainable benefits. Organisations need to invest in developing expertise and software on blockchain programming (lansiti \& Lakhani, 2017) and account for initial mining costs. Simultaneously they may also need to reconfigure their business models and rethink how they provide and charge for services (Iansiti \& Lakhani, 2017; Upadhyay, 2020). While this is likely to incur initial costs, in the end these implementations will benefit both businesses and consumers through sustainability and social protection aligning with the circular economy. Overall, the potential for blockchain to contribute to sustainability and social responsibility, and thereby the circular economy, far outweighs the challenges. Hence, continued investment in blockchain research and its implementation will be extremely valuable for the circular economy.

\section{Conclusions and Future Research}

Through the above narrative review, we demonstrate the intricate link and immense potential of blockchain technology to contribute positively to the circular economy through the sustainability and social responsibility agenda. Blockchain is underpinning applications in various sectors through its capacity to improve track and trace. We analyse blockchain implementation in different sectors, and how this aligns with sustainability and social responsibility concerns. Through our analysis, we show that blockchain contributes to the circular economy through its decentralisation, distributive and high security properties. In particular, our findings show that blockchain technology can contribute to the circular economy by helping to reduce transaction costs, enhance performance and communication along the supply chain, ensure of human rights protection, enhance healthcare 
patient confidentiality and welfare, and reduce carbon footprint. We also identify and evaluate the challenges to blockchain implementation for circular economy, in terms of trust, illegal activities, potential for hacking and the need to address these through suitable legislation and policy development. Furthermore, we acknowledge the potential upfront costs involved in implementing blockchain technology, although we observe that the benefits are likely to far outweigh the challenges.

In this study, we adopted a narrative review approach as we bring together two relatively new fields of study. However given the exponential rate at which research on blockchain is being published, future reviews might benefit from a more quantitative or systematic stance. There is a dearth of articles studying how blockchain technology can contribute to the circular economy. Our article is one of the few to do so and we encourage more research in this arena. There is also a lack of studies focusing on social responsibility within the circular economy model. A gap, we attempt to bridge in this study. We recommend further research on structuring the social responsibility concept within the circular economy model. Moreover, legislation in different countries is likely to influence the development and implementation of blockchain. Future research on potential policy and legislative implications for blockchain and circular economy development will also be valuable. We also recommend further studies on how developing countries with infrastructural challenges can develop circular economies with the help of blockchain technology. 


\section{References}

Beier, G., Ullrich, A., Niehoff, S., Reißig, M., \& Habich, M. (2020). Industry 4.0: How it is defined from a sociotechnical perspective and how much sustainability it includes - A literature review. Journal of Cleaner Production, 259, 120856. https://doi.org/10.1016/j.jclepro.2020.120856

Bengtsson, M., Raza-Ullah, T., \& Vanyushyn, V. (2016). The coopetition paradox and tension: The moderating role of coopetition capability. Industrial Marketing Management, 53, 19-30. https://doi.org/10.1016/j.indmarman.2015.11.008

Bocken, N. M. P., Pauw, I. de, Bakker, C., \& Grinten, B. van der. (2016). Product design and business model strategies for a circular economy. Journal of Industrial and Production Engineering, 33(5), 308-320. https://doi.org/10.1080/21681015.2016.1172124

Catalini, C., \& Gans, J. S. (2016). Some Simple Economics of the Blockchain (Working Paper No. 22952). National Bureau of Economic Research. https://doi.org/10.3386/w22952

De Aguiar, E. J., Faiçal, B. S., Krishnamachari, B., \& Ueyama, J. (2020). A Survey of Blockchain-Based Strategies for Healthcare. ACM Computing Surveys, 53(2), 27:1-27:27. https://doi.org/10.1145/3376915

de Sousa Jabbour, A. B. L., Jabbour, C. J. C., Foropon, C., \& Godinho Filho, M. (2018). When titans meet - Can industry 4.0 revolutionise the environmentally-sustainable manufacturing wave? The role of critical success factors. Technological Forecasting and Social Change, 132, 18-25. https://doi.org/10.1016/j.techfore.2018.01.017

de Sousa Jabbour, A. B. L., Jabbour, C. J. C., Godinho Filho, M., \& Roubaud, D. (2018). Industry 4.0 and the circular economy: a proposed research agenda and original roadmap for sustainable operations. Annals of Operations Research, 270(1-2), 273-286. https://doi.org/10.1007/s10479-018-2772-8

Despeisse, M., Baumers, M., Brown, P., Charnley, F., Ford, S. J., Garmulewicz, A., Knowles, S., Minshall, T. H. W., Mortara, L., Reed-Tsochas, F. P., \& Rowley, J. (2017). Unlocking value for a 
circular economy through 3D printing: A research agenda. Technological Forecasting and Social Change, 115, 75-84. https://doi.org/10.1016/j.techfore.2016.09.021

Dierksmeier, C., \& Seele, P. (2020). Blockchain and business ethics. Business Ethics: A European Review, 29(2), 348-359. https://doi.org/10.1111/beer.12259

Ellen MacArthur Foundation. (2015). Towards a circular economy: Business rationale for an accelerated transition. Report by Ellen MacArthur Foundation.

Ellen MacArthur Foundation. (2019). Artificial intelligence and the circular economy (pp. 1-39). Ellen MacArthur Foundation Report.

Engelhardt, M. A. (2017). Hitching Healthcare to the Chain: An Introduction to Blockchain Technology in the Healthcare Sector. Technology Innovation Management Review, 7(10), 22-34. https://doi.org/10.22215/timreview/1111

Esken, B., Franco-García, M.-L., \& Fisscher, O. A. M. (2018). CSR perception as a signpost for circular economy. Management Research Review, 41(5), 586-604. https://doi.org/10.1108/MRR-022018-0054

Flint, D. (2014). Computers and Internet: Are All Modern Currencies Not Virtual? - The Bitcoin Phenomenon. Business Law Review, 35(2), 60-62.

Geissdoerfer, M., Savaget, P., Bocken, N. M. P., \& Hultink, E. J. (2017). The Circular Economy - A new sustainability paradigm? Journal of Cleaner Production, 143, 757-768.

https://doi.org/10.1016/j.jclepro.2016.12.048

Ghisellini, P., Cialani, C., \& Ulgiati, S. (2016). A review on circular economy: the expected transition to a balanced interplay of environmental and economic systems. Journal of Cleaner Production, 114, 11-32. https://doi.org/10.1016/j.jclepro.2015.09.007

Greenberg, J., \& Mollick, E. (2017). Activist Choice Homophily and the Crowdfunding of Female Founders. Administrative Science Quarterly, 62(2), 341-374.

https://doi.org/10.1177/0001839216678847 
Hens, L., Block, C., Cabello-Eras, J. J., Sagastume-Gutierez, A., Garcia-Lorenzo, D., Chamorro, C., Herrera Mendoza, K., Haeseldonckx, D., \& Vandecasteele, C. (2018). On the evolution of "Cleaner Production" as a concept and a practice. Journal of Cleaner Production, 172, 33233333. https://doi.org/10.1016/j.jclepro.2017.11.082

Iansiti, M., \& Lakhani, K. R. (2017). The truth about blockchain. Harvard Business Review, 119-127. Jesse, N. (2018). Organizational Evolution - How Digital Disruption Enforces Organizational Agility. IFAC-PapersOnLine, 51(30), 486-491. https://doi.org/10.1016/j.ifacol.2018.11.310 Jiang, L., Xie, S., Maharjan, S., \& Zhang, Y. (2019). Blockchain Empowered Wireless Power Transfer for Green and Secure Internet of Things. IEEE Network, 33(6), 164-171. https://doi.org/10.1109/MNET.001.1900008

Lai, R., \& Lee Kuo Chen, D. (2017). Blockchain - From Public to Private. In R. Lai (Ed.), Handbook of Blockchain, Digital Finance, and Inclusion. Academic Press.

Lasi, H., Fettke, P., Kemper, H.-G., Feld, T., \& Hoffmann, M. (2014). Industry 4.0. Business \& Information Systems Engineering, 6(4), 239-242. https://doi.org/10.1007/s12599-014-03344

Li, G., Hou, Y., \& Wu, A. (2017). Fourth Industrial Revolution: technological drivers, impacts and coping methods. Chinese Geographical Science, 27(4), 626-637. https://doi.org/10.1007/s11769-017-0890-x

Loorbach, D., \& Wijsman, K. (2013). Business transition management: exploring a new role for business in sustainability transitions. Journal of Cleaner Production, 45, 20-28. https://doi.org/10.1016/j.jclepro.2012.11.002

Lu, Y. (2017). Industry 4.0: A survey on technologies, applications and open research issues. Journal of Industrial Information Integration, 6, 1-10. https://doi.org/10.1016/j.jii.2017.04.005 Luthra, S., Kumar, A., Zavadskas, E. K., Mangla, S. K., \& Garza-Reyes, J. A. (2020). Industry 4.0 as an enabler of sustainability diffusion in supply chain: an analysis of influential strength of 
drivers in an emerging economy. International Journal of Production Research, 58(5), 15051521. https://doi.org/10.1080/00207543.2019.1660828

MacArthur, D. E., Zumwinkel, K., \& Stuchtey, M. R. (2015). Growth within: A circular economy vision for a competitive Europe. Report of Ellen MacArthur Foundation.

MacInnis, D. J. (2011). A Framework for Conceptual Contributions in Marketing. Journal of Marketing, 75(4), 136-154. https://doi.org/10.1509/jmkg.75.4.136

Mandolla, C., Petruzzelli, A. M., Percoco, G., \& Urbinati, A. (2019). Building a digital twin for additive manufacturing through the exploitation of blockchain: A case analysis of the aircraft industry. Computers in Industry, 109, 134-152. https://doi.org/10.1016/j.compind.2019.04.011

Mathews, J. A., \& Tan, H. (2011). Progress towards a circular economy in China: The drivers (and inhibitors) of eco-industrial initiative. Journal of Industrial Ecology, 15, 435-457.

McGhin, T., Choo, K.-K. R., Liu, C. Z., \& He, D. (2019). Blockchain in healthcare applications: Research challenges and opportunities. Journal of Network and Computer Applications, 135, 62-75. https://doi.org/10.1016/j.jnca.2019.02.027

Moll, J., \& Yigitbasioglu, O. (2019). The role of internet-related technologies in shaping the work of accountants: New directions for accounting research. The British Accounting Review, 51(6), 100833. https://doi.org/10.1016/j.bar.2019.04.002

Murray, A., Skene, K., \& Haynes, K. (2017). The Circular Economy: An Interdisciplinary Exploration of the Concept and Application in a Global Context. Journal of Business Ethics, 140(3), 369-380. https://doi.org/10.1007/s10551-015-2693-2

Nakamoto, S. (2008). Bitcoin: a peer-to-peer electronic cash system.

Narayan, R., \& Tidström, A. (2019). Circular Economy Inspired Imaginaries for Sustainable Innovations. In N. Bocken, P. Ritala, L. Albareda, \& R. Verburg (Eds.), Innovation for Sustainability (pp. 393-413). Springer International Publishing. https://doi.org/10.1007/9783-319-97385-2_21 
Narayan, R., \& Tidström, A. (2020). Tokenizing coopetition in a blockchain for a transition to circular economy. Journal of Cleaner Production, 263, 121437. https://doi.org/10.1016/j.jclepro.2020.121437

O’Dair, M., \& Beaven, Z. (2017). The networked record industry: How blockchain technology could transform the record industry. Strategic Change, 26(5), 471-480. https://doi.org/10.1002/jsc.2147

Omohundro, S. (2014). Cryptocurrencies, smart contracts, and artificial intelligence. Al Matters, 1(2), 19-21. https://doi.org/10.1145/2685328.2685334

Pagoropoulos, A., Pigosso, D. C. A., \& McAloone, T. C. (2017). The Emergent Role of Digital Technologies in the Circular Economy: A Review. Procedia CIRP, 64, 19-24. https://doi.org/10.1016/j.procir.2017.02.047

Purvis, B., Mao, Y., \& Robinson, D. (2019). Three pillars of sustainability: in search of conceptual origins. Sustainability Science, 14(3), 681-695. https://doi.org/10.1007/s11625-018-0627-5

Püttgen, F., \& Kaulartz, M. (2017). Insurance 4.0: The use of blockchain technology and of smart contracts in the Insurance Sector. ERA Forum, 18(2), 249-262. https://doi.org/10.1007/s12027-017-0479-y

Radanović, I., \& Likić, R. (2018). Opportunities for Use of Blockchain Technology in Medicine. Applied Health Economics and Health Policy, 16(5), 583-590. https://doi.org/10.1007/s40258-018$0412-8$

Rozario, A. M., \& Vasarhelyi, M. A. (2018). Auditing with Smart Contracts. The International Journal of Digital Accounting Research, 18, 1-27. https://doi.org/10.4192/1577-8517-v18_1

Saberi, S., Kouhizadeh, M., Sarkis, J., \& Shen, L. (2019). Blockchain technology and its relationships to sustainable supply chain management. International Journal of Production Research, 57(7), 2117-2135. https://doi.org/10.1080/00207543.2018.1533261 
Schmidt, C. G., \& Wagner, S. M. (2019). Blockchain and supply chain relations: A transaction cost theory perspective. Journal of Purchasing and Supply Management, 25(4), 100552. https://doi.org/10.1016/j.pursup.2019.100552

Schmitz, J., \& Leoni, G. (2019). Accounting and Auditing at the Time of Blockchain Technology: A Research Agenda. Australian Accounting Review, 29(2), 331-342. https://doi.org/10.1111/auar.12286

Schulte, U. G. (2013). New business models for a radical change in resource efficiency. Environmental Innovation and Societal Transitions, 9, 43-47. https://doi.org/10.1016/j.eist.2013.09.006

Schumacher, A. (2017). Schumacher, A. 2017. Blockchain \& Healthcare-2017 Strategy Guide. Axel Schumacher.

Seele, P. (2018). Let Us Not Forget: Crypto Means Secret. Cryptocurrencies as Enabler of Unethical and Illegal Business and the Question of Regulation. Humanistic Management Journal, 3(1), 133-139. https://doi.org/10.1007/s41463-018-0038-x

Sousa-Zomer, T. T., Magalhães, L., Zancul, E., \& Cauchick-Miguel, P. A. (2017). Lifecycle Management of Product-service Systems: A Preliminary Investigation of a White Goods Manufacturer. Procedia CIRP, 64, 31-36. https://doi.org/10.1016/j.procir.2017.03.041

Spring, M., \& Araujo, L. (2017). Product biographies in servitization and the circular economy. Industrial Marketing Management, 60, 126-137. https://doi.org/10.1016/j.indmarman.2016.07.001

Sun, M., \& Zhang, J. (2020). Research on the application of block chain big data platform in the construction of new smart city for low carbon emission and green environment. Computer Communications, 149, 332-342. https://doi.org/10.1016/j.comcom.2019.10.031

Swan, M. (2015). Blockchain: blueprint for a new economy (First edition). O’Reilly.

Szabo, N. (1997). Formalizing and securing relationships on public networks. First Monday, 2(9). http://firstmonday.org/ojs/index.php/fm/article/view/548/469 
Tang, Q., \& Tang, L. M. (2019). Toward a Distributed Carbon Ledger for Carbon Emissions Trading and Accounting for Corporate Carbon Management. Journal of Emerging Technologies in Accounting, 16(1), 37-46. https://doi.org/10.2308/jeta-52409

Torraco, R. J. (2005). Writing Integrative Literature Reviews: Guidelines and Examples. Human Resource Development Review, 4(3), 356-367. https://doi.org/10.1177/1534484305278283

Treleaven, P., Gendal Brown, R., \& Yang, D. (2017). Blockchain Technology in Finance. Computer, 50(9), 14-17. https://doi.org/10.1109/MC.2017.3571047

United Nations. (2012). The future we want. Resolution adopted by the general assembly on 27 July 2012 (A/RES/66/288). United Nations.

United Nations. (2015). UN (2015) Transforming our world: the 2030 Agenda for sustainable development. Resolution adopted by the general assembly on 25 September 2015 (A/RES/70/1). United Nations.

Upadhyay, A., Mukhuty, S., Kumari, S., Garza-Reyes, J. A., \& Shukla, V. (In Press). A Review of Lean and Agile Management in Humanitarian Supply Chains: Analysing the Pre-Disaster and PostDisaster Phases, and Future Directions. Production Planning \& Control: The Management of Operations.

Upadhyay, N. (2020). Demystifying blockchain: A critical analysis of challenges, applications and opportunities. International Journal of Information Management, 54, 102120. https://doi.org/10.1016/j.ijinfomgt.2020.102120

Vatankhah Barenji, A., Li, Z., Wang, W. M., Huang, G. Q., \& Guerra-Zubiaga, D. A. (2020). Blockchainbased ubiquitous manufacturing: a secure and reliable cyber-physical system. International Journal of Production Research, 58(7), 2200-2221. https://doi.org/10.1080/00207543.2019.1680899

Venkatesh, V. G., Kang, K., Wang, B., Zhong, R. Y., \& Zhang, A. (2020). System architecture for blockchain based transparency of supply chain social sustainability. Robotics and ComputerIntegrated Manufacturing, 63, 101896. https://doi.org/10.1016/j.rcim.2019.101896 
Wang, Y., \& Kogan, A. (2018). Designing confidentiality-preserving Blockchain-based transaction processing systems. International Journal of Accounting Information Systems, 30, 1-18. https://doi.org/10.1016/j.accinf.2018.06.001

Wu, X., \& Lin, Y. (2019). Blockchain recall management in pharmaceutical industry. Procedia CIRP, 83, 590-595. https://doi.org/10.1016/j.procir.2019.04.094

Xu, M., Chen, X., \& Kou, G. (2019). A systematic review of blockchain. Financial Innovation, 5(1), 27. https://doi.org/10.1186/s40854-019-0147-z

Xue, B., Chen, X. P., Geng, Y., Guo, X. J., Lu, C. P., \& Zhang, Z. L. (2010). Survey of officials' awareness on circular economy development in China: Based on municipal and county level. Resources, Conservation and Recycling, 54, 1296-1302.

Yue, X., Wang, H., Jin, D., Li, M., \& Jiang, W. (2016). Healthcare Data Gateways: Found Healthcare Intelligence on Blockchain with Novel Privacy Risk Control. Journal of Medical Systems, 40(10), 218. https://doi.org/10.1007/s10916-016-0574-6 